\title{
EFFECT OF SPECIMEN SIZE ON STRENGTH RELIABILITY OF FIBER REINFORCED COMPOSITE
}

\author{
Yun $\mathrm{Lu}^{1}$, Hideharu Fukunaga ${ }^{2}$ \\ ${ }^{I}$ Faculty of Engineering, Chiba University, 1-33, Yayoi-cho, Inage-ku, Chiba, 263-8522, \\ Japan; E-mail: luyun@faculty.chiba-u.jp, ${ }^{2}$ Kure College of Technology, Institute of National \\ Colleges, Japan, 2-2-11, Agaminami, Kure, Hiroshima, 737-8506, Japan
}

Abstract: A tensile test was carried out for continuous $\mathrm{SiC}$ fiber reinforced aluminum matrix composites ( $\mathrm{SiC} / \mathrm{Al}$ ), which have different specimen width and length. A Monte Carlo simulation using finite element method was also performed with changing the width and length of composite model. The size effect of strength reliability was examined and discussed. The results showed that composite strength does not appear to have a large change but its reliability is improved with increasing in width of the composite specimen. Also, the composite strength decreases, but Weibull shape parameter has an increase tendency with increasing the gauge length. Effect of specimen width and length on composite strength and its reliability is dependent on the both factors, that is, the incidence of accumulating fracture process, the mean fiber strength and the strength scatter of weaker fibers. The accumulation of the fracture process is related to the fiber stress distribution in the composite.

Key words: fiber reinforced metal, SiC/Al composite, tensile test, Monte Carlo simulation, specimen width, gauge length, strength reliability, size effect.

\section{INTRODUCTION}

It was clarified that both strength and its reliability of single or bundle ceramic fiber have an obvious size effect ${ }^{1-4}$. According to these studies, the strength decreases and its scatter becomes wide with the gauge length of single ceramic fiber ${ }^{3}$. In the case of ceramic fiber bundle, the strength and its

Please use the following format when citing this chapter:

Lu, Yun, Fukunaga, Hideharu, 2006, in International Federation for Information

Processing (IFIP), Volume 207, Knowledge Enterprise: Intelligent Strategies In Product Design, Manufacturing, and Management, eds. K. Wang, Kovacs G., Wozny M., Fang M., (Boston: Springer), pp. 767-779. 
scatter go down and finally the both converge to the value from the Coleman theory with increase of the fiber number in a bundle ${ }^{1,4}$. Therefore, strength and its reliability of the fiber reinforced metal composite fabricated with use of ceramic fibers and metal matrix can be understood to have the size effect. It is very important to investigate the size effect of composite strength and its reliability from the view point of application for structural materials. Recently, it was reported that the strength has large size effect for fiber reinforced plastic composites ${ }^{5-6}$.

In this study, a unidirectional SiC/Al composite having a constant fiber volume fraction was produced by hot press method. A tensile test was carried out for $\mathrm{SiC} / \mathrm{Al}$ composite specimens with different width or gauge length. The size effect of composite strength and its reliability was examined. Meanwhile, a Monte Carlo simulation using finite element method was performed. The size effect was discussed taking fracture process or stress distribution in the composite into consideration.

\section{SPECIMEN AND EXPERIMENTAL}

A1050 pure aluminum plate with a thickness of $0.3 \mathrm{~mm}$ was used as the matrix material. $\mathrm{SiC}$ fiber manufactured by a chemical vapor deposition method, obtained from Textron Specialty Materials Company (type: SCS-2, diameter: $140 \mu \mathrm{m}$ ), was used as the reinforcement. SiC/Al composite was fabricated by hot press process method. Firstly an aluminum plate was cut into $30 \times 70 \mathrm{~mm}^{2}$ pieces which were then annealed at $573 \mathrm{~K}$ for $30 \mathrm{~min}$ in air. In order to arrange the fibers on the aluminum pieces orderly, U-shaped grooves with an interspace of $0.5 \mathrm{~mm}$ were made on one side of the aluminum pieces by pressing the stainless steel wires (with a diameter of $140 \mu \mathrm{m}$ ) into the piece. Secondly, SiC fibers, which were cut into a length of $70 \mathrm{~mm}$ from the obtained successive SiC fiber, were put into the grooves on the surface of each aluminum piece, which was then overlapped by another aluminum piece. Finally, this one layer $\mathrm{SiC} / \mathrm{Al}$ perform was hot pressed into the composite by the process of temperature $893 \mathrm{~K}$, under the pressure of $56 \mathrm{MPa}$ and holding time $40 \mathrm{~min}$ in air. The prepared SiC/Al composites have a dimension about $70 \mathrm{~mm}$ length and $0.55 \mathrm{~mm}$ thickness. Fiber volume fraction is about $4.7 \%$. In the case of changing the specimen width, tensile specimens were cut out from $\mathrm{SiC} / \mathrm{Al}$ composite, changing the specimen width and making the fibers to be being 10,15, 20 and 30 in the cross section. Tabs of aluminum plate were set to both the ends and the gauge length was a constant, $20 \mathrm{~mm}$ for all the composite specimens. In the case of changing the gauge length, tensile specimens with gauge lengths of 5, 10, 20, 30 and $40 \mathrm{~mm}$, and a constant fiber number, 10 in the cross section were prepared by locating the tabs of aluminum plate. 
In addition, to understand the fiber strength in the $\mathrm{SiC} / \mathrm{Al}$ composite, the fibers were extracted from $\mathrm{SiC} / \mathrm{Al}$ composite by dissolving the aluminum matrix with $10 \% \mathrm{NaOH}$ water solution, and then a tensile test was carried out for the fibers. Tabs of aluminum plate were set to both the ends of all tensile specimens.

The tensile test was completed in a screw driven tester (Shimadzu, AG$5000 \mathrm{ES}$ ). All tests were done at room temperature with a constant crosshead speed of $0.5 \mathrm{~mm} / \mathrm{min}$. The load was recorded as a function of time using a load-time recorder. For each specimen width and gauge length, the numbers of tensile specimens for SiC/Al composite were over 40, but extracted SiC fiber were over 50, respectively. The strength of the composite and the extracted $\mathrm{SiC}$ fiber was evaluated by using 2 parameters Weibull distribution function. Figure 1 shows a SEM image of cross section microstructure of $\mathrm{SiC} / \mathrm{Al}$ composite produced in the present study. It can be seen that the $\mathrm{SiC}$ fibers arrange in almost the same interspace orderly and a good compound between the fiber and the matrix is obtained.
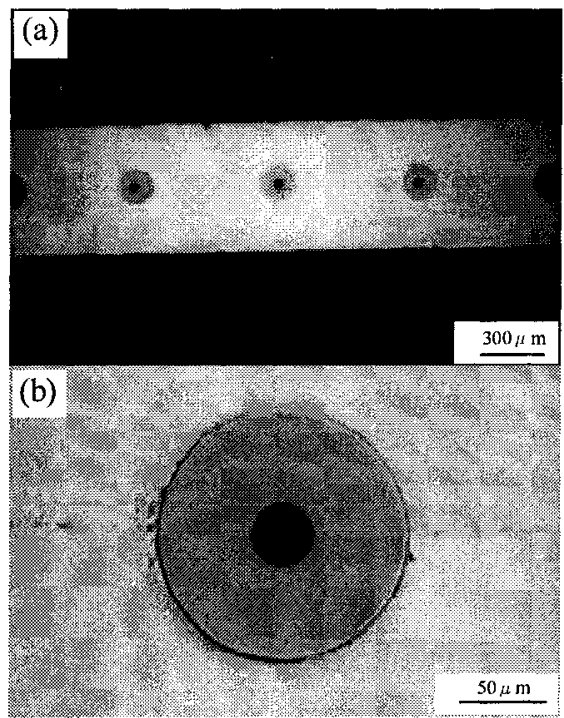

Figure 1. SEM image of cross section microstructure of SiC/Al composite.

\section{MODEL AND TECHNIQUE OF THE SIMULATION}


A Monte-Carlo simulation using elastic-plastic finite element method for evaluating the tensile strength and its scatter of the unidirectional $\mathrm{SiC} / \mathrm{Al}$ composite was performed. The finite element model and mesh in the present study are shown as in Fig.2. This model is built on a monolayer plate of unidirectional fiber reinforced metal including 3, 5, 10, 15, 20 and 25 fibers. The two one-dimensional line elements, which represent the reinforcing fibers, are incorporated into two sides along the y-axis of a 4-node isoparametric element (plane stress), which represents the metal matrix. The

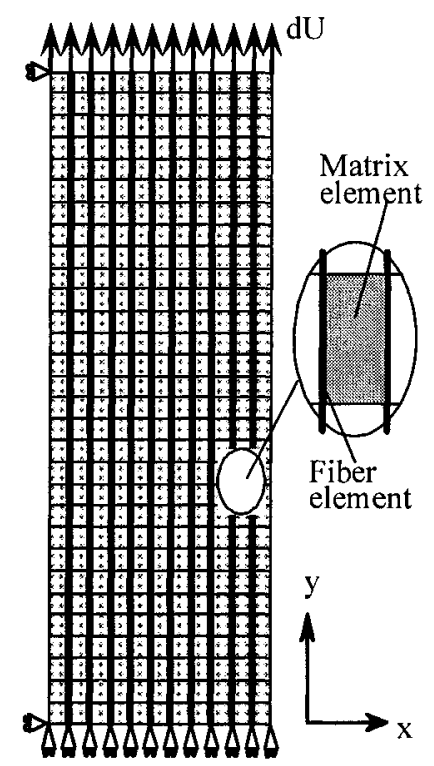

Figure 2. Finite element model (including 10 fibers).

model is divided into 30 elements in y-axis, and has $\mathrm{N}_{\mathrm{f}} \times 30$ fiber elements, $\left(\mathrm{N}_{\mathrm{f}}+1\right) \times 30$ matrix elements and $\left(\mathrm{N}_{\mathrm{f}}+2\right) \times 31$ nodes. Where, $\mathrm{N}_{\mathrm{f}}$ shows fiber number in the model. $y$-axis of nodes in the lower side is fixed, but displacement increment is given along $y$-axis of nodes in the upper side. The strength, $\sigma_{\mathrm{fi}}$ of fiber elements is given by Weibull random number, using the inverse function of Weibull distribution as equation 1 .

$$
\sigma_{\mathrm{fi}}=\sigma_{0}\left\{\left(\mathrm{~L}_{0} / \Delta \mathrm{X}\right) \ln [1 /(1-\mathrm{Z})]\right\}^{1 / \mathrm{mf}}
$$

Where, $\sigma_{0}$ and $m_{f}$ show Weibull scale and shape parameters of the fiber strength, respectively. $L_{0}$ is a standard length. $\Delta X$ corresponds to the element length along the $y$-axis. $Z$ is random number in $(0,1)$ which was produced by the multiplicative congruential method. In the present study, $\sigma_{0}$ and $m_{f}$ ' were substituted with ones of the $\mathrm{SiC}$ fiber, which were obtained by a tensile test 
of the extracted fiber from $\mathrm{SiC} / \mathrm{Al}$ composite. Also, $\mathrm{L}_{0}$ was substituted with $10 \mathrm{~mm}$, which is the gauge length of a tensile test for the extracted fiber. Where, the fiber elastic modulus is maker's data. The parameters obtained by a tensile test of A1050 pure aluminum were used as mechanical property parameters of the matrix in this simulation. The parameters of the $\mathrm{SiC}$ fiber and the matrix used in the present simulation are shown in Table 1.

Table 1. Material parameters for the present simulation.

\begin{tabular}{lc}
\hline Fiber & \\
Diameter (mm) & 0.14 \\
Elastic module (GPa) & 421.7 \\
Strength (GPa) & 4.15 \\
Weibull scale parameter (GPa) & 4.15 \\
Weibull shape parameter & 4.42 \\
& \\
Matrix & \\
Elastic module (GPa) & 70 \\
Yield stress (MPa) & 23.2 \\
Work hardening coefficient (MPa) & 176 \\
Work hardening exponent & 0.27 \\
Poisson's ratio & 0.33 \\
& \\
Composite & \\
Gauge length (mm) & $20,4-100$ \\
Element length (mm) & $0.67,2.0$ \\
Thickness (mm) & 0.6 \\
Distance between fibers (mm) & 0.5 \\
Fiber number & $3-25$ \\
Fiber volume fraction (\%) & 4.7 \\
& \\
\hline
\end{tabular}

Furthermore, for exactly estimating reasonably each element stress increment necessary to the fiber breaking or the matrix yielding, the $r_{\min }$ method is applied to the simulation procedure ${ }^{7}$. The fibers are regarded as elastic state but the matrix is assumed to be elastic-plastic. Fiber element is considered to break when the stress, which is along the axial direction of the fiber, reaches the strength of that fiber element. After a fiber element breaks, the stiffness is changed to zero. The axial load, which was loading on that fiber element, is decreased to zero by dividing the load into 20 times, which was decided by pre-simulation. When composite stress decreases below $80 \%$ of the maximum stress, the composite is considered to be failure, and then the simulation is finished. This simulation was carried out 50 times for supposed each specimen width, respectively. The flow chart of the present simulation is shown in Fig.3. For the composite with different gauge length, 
the above simulation was carried out by changing the gauge length from $4 \mathrm{~mm}$ to $100 \mathrm{~mm}$, but using a model with a constant fiber number of 10 fibers and the same parameters as listed in Table 1.

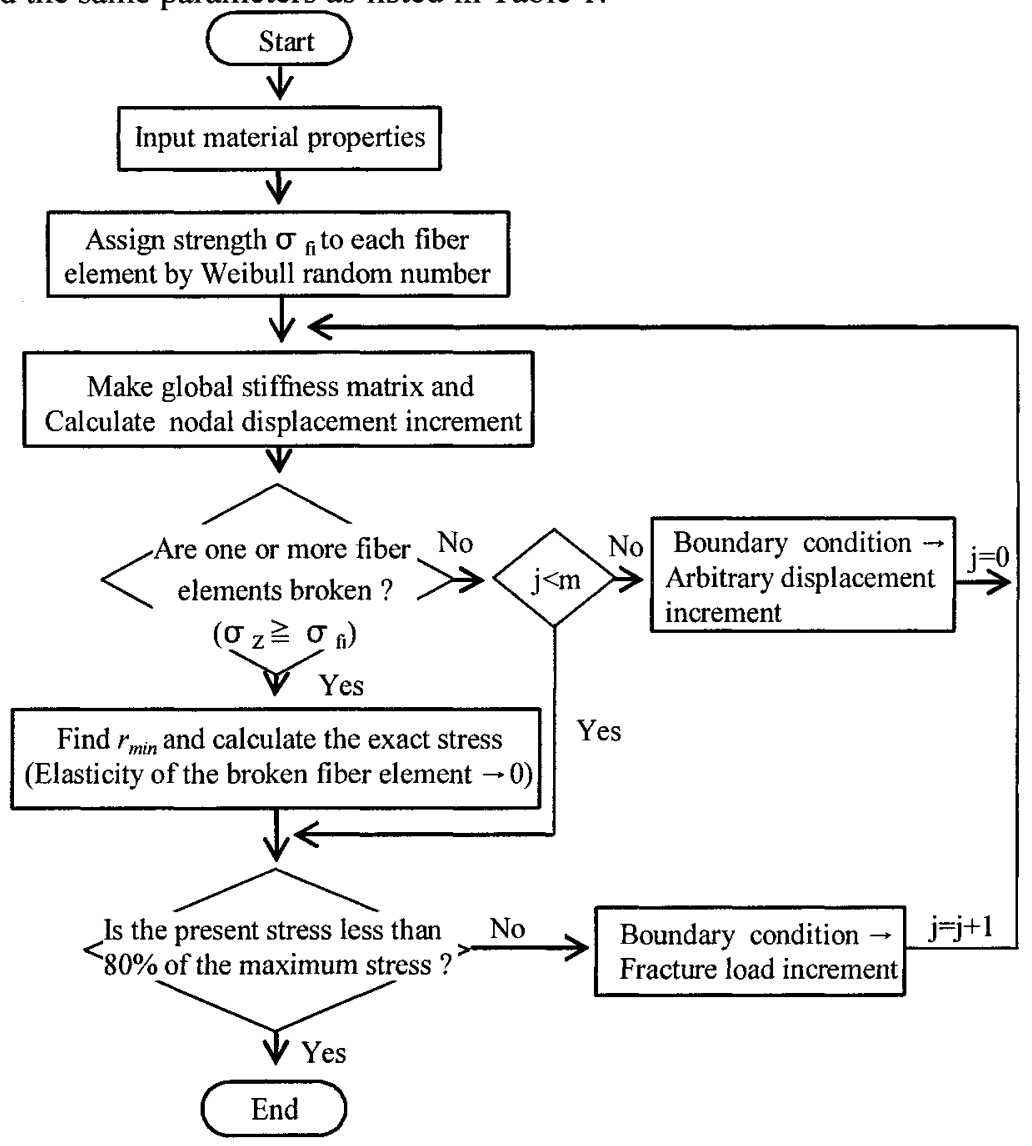

Figure 3. Flow chart of the present simulation.

\section{RESULTS AND DISCUSSION}

Tensile stress-strain curves obtained from tensile test and the present simulation for $\mathrm{SiC} / \mathrm{Al}$ composite are shown in Fig.4. It can be seen that the slope of the curves has a change owing to yielding of the matrix. Till the maximum stress (composite strength), there are abrupt drops of the stress at some locations on the curves. The abrupt stress drops are considered to originate in the fibers breaking in the composite. Also, it is understood from the strain-stress curves to be possible to simulate the tensile behavior of the 
composite by the present simulation. Relationship between specimen width against composite strength and Weibull shape parameter is shown in Fig. 5. The specimen width was represented by the fiber number in the specimen. Composite strength almost does not change with increasing the specimen width (see Fig.5 (a)). But Weibull shape parameter $m$ goes up; that is, the reliability is improved with increasing the specimen width (see Fig.5 (b)).

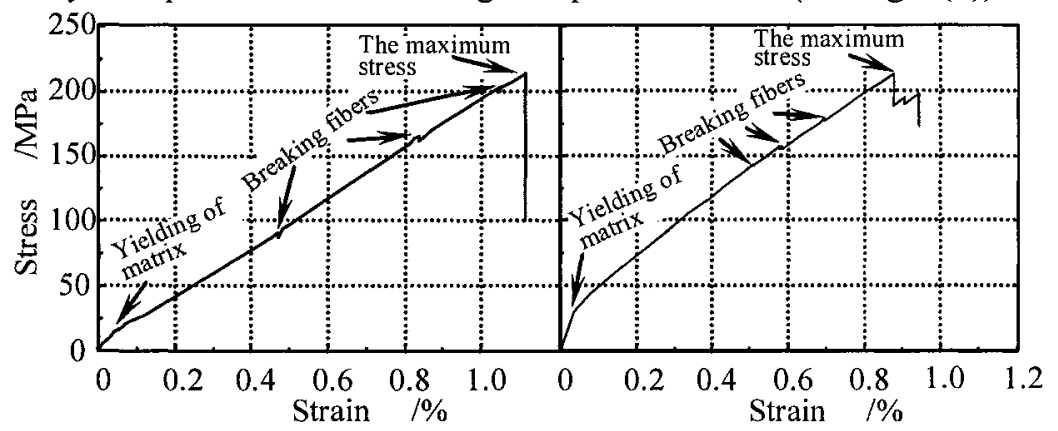

(a) Experiment

(b) Simulation

Figure 4. Stress-strain curves of $\mathrm{SiC} / \mathrm{Al}$ composite with 15 fibers and $20 \mathrm{~mm}$ gauge length

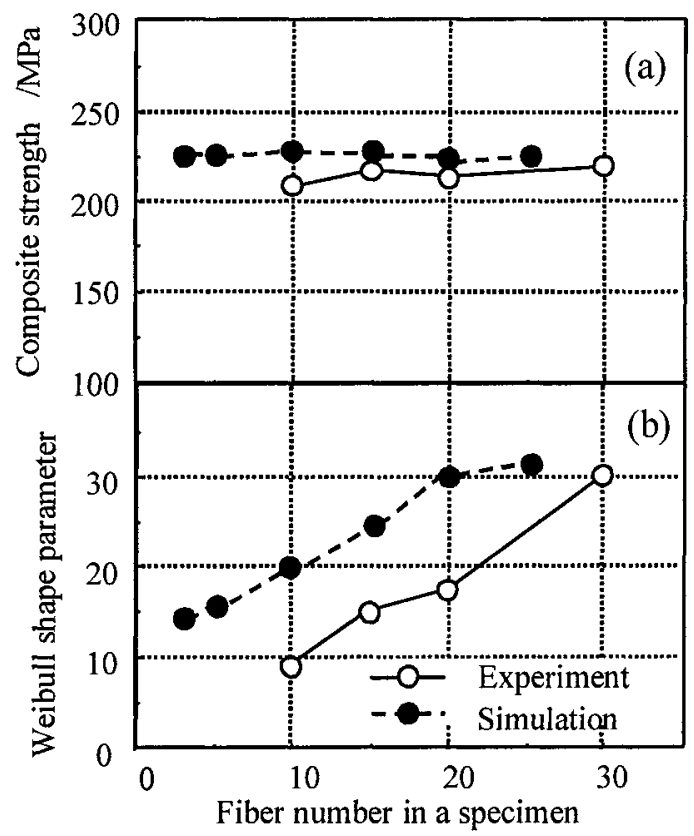

Figure 5. Relationship between specimen width against composite strength and Weibull shape parameter. 
Generally, when a composite is loaded, the relatively weak fibers break at first. Till the maximum stress, fiber breaking occurs many times as shown in Fig.4. To understand the relationship between the composite strength, its reliability and the fracture process, the accumulated broken fiber was counted by reading out the number of breaking fiber element till the maximum stress during loading the composites in the present simulation. Relationship between the average count of broken fiber against composite strength and Weibull shape parameter is shown in Fig. 6. The strength does not display any change, but Weibull shape parameter increases with the average count. It hints that the reliability is related to fracture process of the composite.

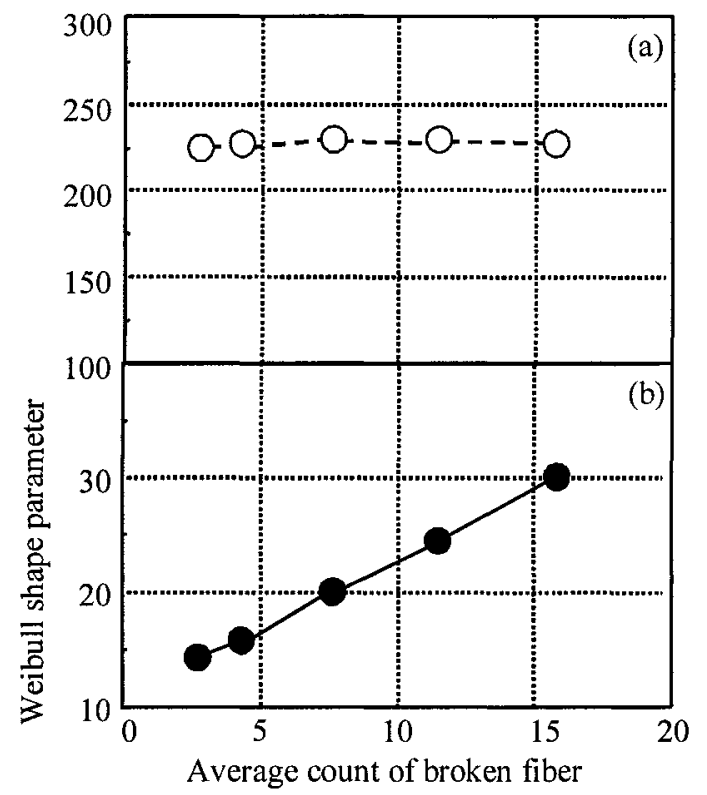

Figure 6. Relationship between average count against composite strength and Weibull shape parameter from the simulation.

Furthermore, in order to discuss the effect of the accumulation of breaking fiber on composite strength and its reliability, the fiber stress in the composite was read out in the order of broken fiber on every simulation for each the specimen width. Then average fiber stress and Weibull shape parameter were calculated by Weibull distribution for each fiber breaking. Figure 7 shows relationship between the order number of broken fiber against the fiber stress and Weibull shape parameter. The fiber stress and Weibull shape parameter show a increase tendency with the order number for each width. That is, the more the accumulating fiber fracture is, the higher fiber stress needed for the fracture is, and the lower the fiber stress scatter is. Therefore, the more the accumulating fiber fracture is, it becomes 
more obvious that composite strength is controlled by the fiber, which has a high strength and low strength scatter.

On the other hand, it is easy to understand that number of weaker fiber increases with increasing the width, that is, fiber number in the specimen. Figure 8 shows relationship between fiber number in SiC/Al composite against fiber strength and Weibull shape parameter of the weakest fiber element which were the first broken fiber element shown as order number 1 in Fig.7. The weakest fiber strength goes down extensively but Weibull shape parameter just only decreases gently. From above results, it can be understood that effect of specimen width on composite strength and its reliability is dependent on both factors, that is, the one is the accumulating of fracture process and the other is fiber strength and its scatter of the weaker fibers in the composite. It can be explained from both the above factors why composite strength almost does not change, but Weibull shape parameter $m$ of the strength goes up with increasing the specimen width in the present study.

Finally, in order to investigate the relationship between stress distribution in the composite specimen and specimen width, stress distribution was calculated when making only the center fiber element to break. In the calculation model, it is supposed that there are 5, 9, 19 and 31

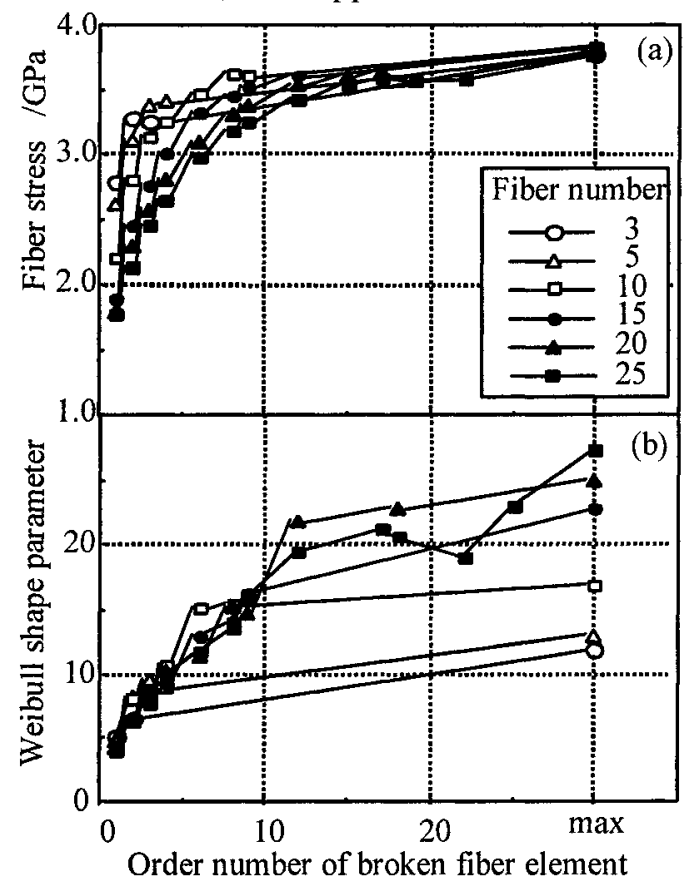


Figure 7. Order number of broken fiber vs fiber stress and Weibull shape parameter from the simulation.

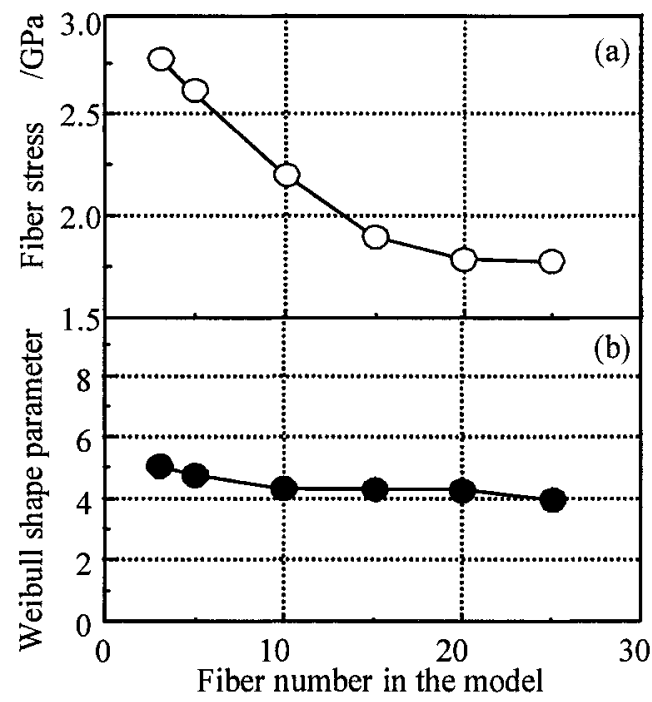

Figure 8. Fiber number in the composite model vs stress and Weibull shape parameter of the weakest fiber from the simulation.

fibers. To calculate the stress distribution after fiber breaking accurately, the extracted fiber strength, $4.15 \mathrm{GPa}$ shown in Table 1 was given to the central fiber element, and enough large strength value (100GPa) was given to the other fiber elements in this model. Thus, only the central fiber element breaks but the other fiber elements do not break when the composite is loaded. The fiber stress distribution along the broken fiber and the vertical direction of the fiber were calculated. Where the fiber stress distribution is represented by stress concentration factor, which is calculated out by dividing stresses of all fiber element in the fiber stress at the lowest fiber elements in the model. The results are illustrated in Fig. 9. From this figure, stress concentration factor along broken fiber almost is not different for each case of the width. However, stress concentration factor along the vertical direction of the fiber goes up with decreasing the width. Thus, the fiber breaking is spread or continued easily to other fibers due to the large stress concentration factor when the width is small. It decreases the accumulating of the fracture process in the case of narrow composite specimen. 


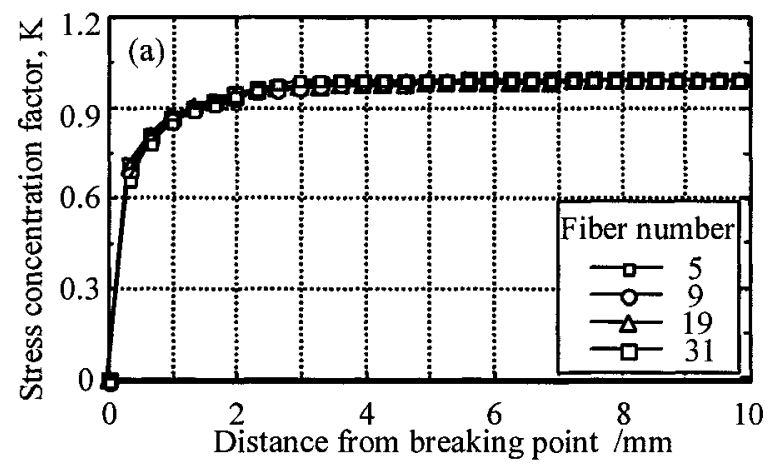

(a) Distribution of fiber stress along broken fiber from breaking point.

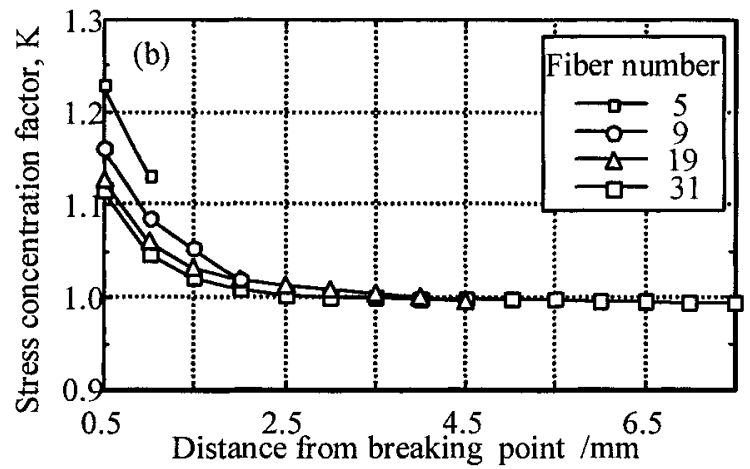

(b) Distribution of fiber stress along the vertical direction of the fiber from breaking point.

Figure 9. Distribution of fiber stress along the broken fiber and vertical direction of the fibers for the composite with different width.

Figure 10 shows strength, Weibull shape parameter and average count of broken fiber for the composite with different gauge length from the present experiment and simulation. The composite strength decreases, but Weibull shape parameter has an increase tendency with increasing the gauge length. It is understood to relate with decrease of reinforced fiber strength with the specimen length and the accumulation of breaking fiber in the composite showed as in Fig.10(c). Also, the fiber stress distribution along the broken fiber and the vertical direction of the fiber were calculated for the composite with different gauge length as the case of the different width. The results are illustrated in Fig. 11. From this figure, stress concentration factor along broken fiber almost is not different for each case of the gauge length. However, stress concentration factor along the vertical direction of the fiber goes up with increasing the gauge length to $22 \mathrm{~mm}$, but then thereafter, does 
not have change. That may be related to the end effect when the gauge length is shorter than $22 \mathrm{~mm}$.

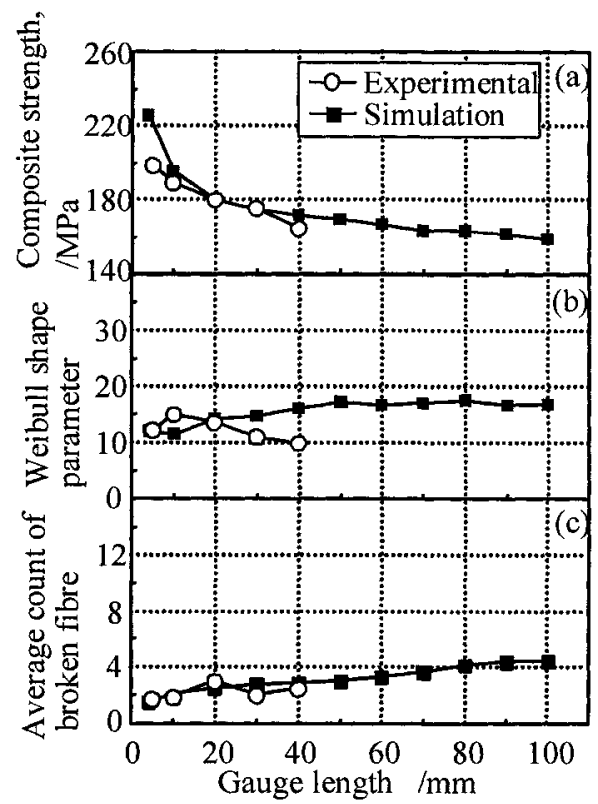

Figure 10. Relationships between gauge length and (a) composite strength, (b) Weibull shape parameter of the strength, and (c) average count of broken fiber for the case of different gauge length.

\section{CONCLUSION}

1) Composite strength almost does not change, but Weibull shape parameter of the strength goes up with increasing the specimen width. It indicates that the reliability is improved with increasing the specimen width. In the case of the composite with different gauge length, the composite strength decreases, but Weibull shape parameter has an increase tendency with increasing the gauge length.

2) Effect of specimen width on composite strength and its reliability is dependent on both factors, that is, (a) the accumulating of the fracture process and (b) strength and its scatter of the weaker fibers in the composite.

3) The accumulating of the fracture process is related to the fiber stress distribution in the composite. 

Composite

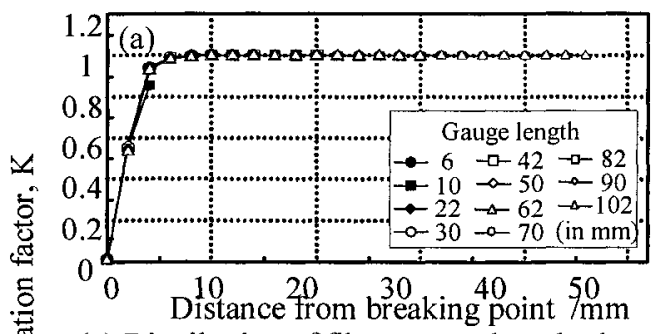

(a) Distribution of fiber stress along broken fiber from breaking point.

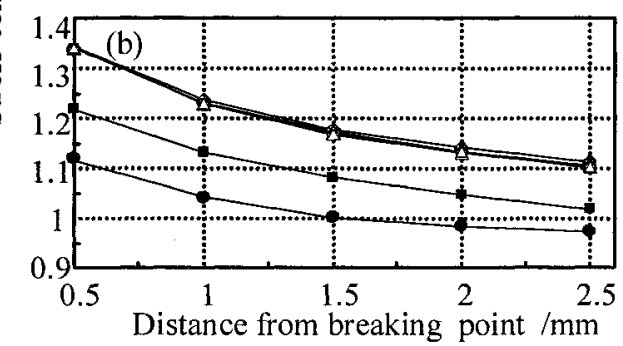

(b) Distribution of fiber stress along the vertical direction of the fiber from breaking point.

Figure 11. Distribution of fiber stress along the broken fiber and vertical direction of the fibers for the composite with different gauge length.

\section{REFERENCES}

1. B. D. Coleman, (1958), J. Mech. Phys. Solids, Vol.7, 60-70.

2. M. G. Bader and A. M. Priest, (1982), Progress in science and engineering of composite, T. Hayashi, K. Kawata \& S. Umekawa, Ed., ICCM-IV, Tokyo, pp.1129-1136.

3. Yun LU, M. Hirohashi and O. Yashima, (1996),Proceeding of the 73rd JSME spring annual meeting, Vol.II, pp.280-281.

4. Yun LU, M. Hirohashi T. Nomiya and Y. Araoka, (1995), Proceeding of the 72nd JSME spring annual meeting, Vol.II, pp.250-251.

5. Pickering K. L and Murray T. L., (1999), Composites Part A, Vol.30A, No.8, 1017-1021.

6. Wisnom M. R., (1999), Compos. Sci. Technol., Vol.59, No.13, 1937-1957.

7. K. Washizu, H. Miyamoto, Y. Yamata, Y. Yamamoto and T. Kawai, (1992), Handbook of Finite Element Method, Baifukan Ltd., Japan. 